\title{
Endemic Typhus Fever
}

National Cancer Institute

\section{Source}

National Cancer Institute. Endemic Typhus Fever. NCI Thesaurus. Code C84688.

A bacterial infection caused by Rickettsia typhi or Rickettsia felis. It is transmitted to humans from infected rat fleas. Symptoms include fever, headache, joint and muscle pain, and weakness. 